\title{
VIDEO IMAGE SELECTION STUDIES OF GRANULES, PORES, AND PENUMBRAL FLOWS NEAR A LARGE SUNSPOT
}

\author{
HAROLD ZIRIN and HAIMIN WANG
}

Big Bear Solar Observatory, California Institute of Technology, Pasadena, CA 91125, U.S.A.

(Received 15 August, 1988; in revised form 19 September, 1988)

\begin{abstract}
An excellent high-resolution movie in the green continuum was produced by shift-and-add treatment of two 60-min videotapes obtained at the Big Bear Solar Observatory. We have studied the digitized images by direct measurement, cross-correlation techniques, and correlation tracking. The seeinglimited resolution was about 0.3 arc sec.

While the cross-correlation lifetime for granules is about five minutes, we find that actually tracking the growth and decay of a granule gives lifetimes from 10 to 22 minutes, the longest lifetimes pertaining to the largest granules. The longer lifetime comes from tracking the granule while it undergoes large changes in size and shape, while the cross-correlation lifetime is just the time in which it grows by a factor two. All the granules followed began as small elements, grew to some size, and either faded $(88 \%)$, exploded $(2 \%)$ or were hit by an exploding granule $(10 \%)$. The major variation in granule structure appears to be due to substantial variations in the dark lanes, which often double in width.

The granulation shows the typical exploding granule behavior; we find the probability that any granule will be affected by an exploding granule during its lifetime to be $10 \%$. We also observed a larger scale explosion covering about 10 granules. This 'explosion' was marked by rapid $\left(1 \mathrm{~km} \mathrm{~s}^{-1}\right)$ outward flux of the granules.

We tracked the development of six small pores, one of which could be followed for two hours. The latter showed four maxima of absorption separated by about $30 \mathrm{~min}$ each, virtually disappearing in between. Another was observed to form in about $20 \mathrm{~min}$, but no changes occur in less than granule lifetime.

We confirm the inflow in penumbral fibrils observed by Muller. The inflow velocity is about $0.5 \mathrm{~km} \mathrm{~s}^{-1}$, and all bright spots disappear into the umbra. The inflow which affects bright and dark features in the penumbral fibrils, is also observed in the smaller spots. We surmise that the Evershed flow is limited to the areas between the bright fibrils. We confirm granular outflow outside the penumbra.
\end{abstract}

\section{Introduction}

There have been many studies of the solar granulation (see reviews by Bray, Loughead, and Durrant, 1984; and Muller, 1985). The granules have a diameter in the range of 1 " to 2 ", lifetime 6 to $16 \mathrm{~min}$. The morphology of granules have been discussed in detail by many authors (Kitai and Kawaguchi, 1979; Kawaguchi, 1980; Oda, 1984; Roudier and Muller, 1987). In recent years interest in high-resolution observations has brought new data, and white light films obtained by the Solar Optical Universal Polarimeter (SOUP) instrument on Spacelab 2 (Title et al., 1986, Simon et al., 1988) have produced new data on the lifetime, evolution, and proper motions of granules.

In this paper we study the result of the application of modern ground-based optical techniques to the problem. The data are digitally selected and processed from highresolution videotapes obtained at the Big Bear Solar Observatory. While much of the data confirms results obtained by the authors mentioned above, considerable new information is found on these tapes, and the technique simply requires waiting for good seeing, which is slightly more frequent than spaceflight opportunities. We will show 
results on the lifetime of granules, exploding granules, fast changing pores, proper motion of granules and the penumbral flow.

\section{Data}

On July 11, 1986 we obtained high-quality videotapes for two hours on 0.75 in 'U-matic' videotape with the $65-\mathrm{cm}$ vacuum reflector. Two regions near a sunspot were observed for 60 min each. Region 1 was a granular area near a large symmetric sunspot and included small pores; it was observed from $16: 45$ to $17: 47$ UT. Region 2 was centered on the sunspot and observed from 17:51 to 18:52 UT. We used an RCA CCD camera with a Schott VG-9 green filter yielding about 400 lines resolution (our current technique employs a filter at $4600 \AA$, where contrast is higher, a better CCD, and super-VHS tape, which permits higher resolution and records for two hours). The field of view was about $45^{\prime \prime} \times 40^{\prime \prime}$, giving a pixel resolution of about $0.1 \mathrm{arc}$ sec. Dust specks on the CCD faceplate are invariably sharper and darker than solar structures; hence, the data is limited by seeing and telescope Modulation Transfer Function (MTF) to about 0.3 arc sec.

The images obtained were affected by variable seeing and tracking. Therefore, we picked out the best frames in each $10 \mathrm{~s}$ interval (comprising 360 frames for each 60 -min tape). These frames were selected and digitized with our Megavision image processor. The images were registered on our MicroVAX using a batch program written by $\mathrm{Dr}$ Bruce Popp. These images were then displayed on a video screen and recorded on film. In addition, a 56-frame movie was stored in the computer, where it could be viewed at any desired rate. These films enable us to watch the evolution of elements almost free of seeing effects.

Since the limiting visibility of the granules is strictly determined by the system and atmospheric MTF, we cannot say what the true contrast of the smallest dark lanes is. However, we measured the brightness distribution for the record. The images were calibrated by a transmission 0.47 filter in the corner of the image, and the system is known to be linear. We made histograms of pixel brightness distribution and found it quite uniform from frame to frame and point to point in the frame (always taking the best frames). The histogram was well-fitted by a gaussian of standard deviation 0.12 times the mean intensity. The peak to darkest ratio was 2.6. By partitioning the image until we had selected those areas that looked like dark lanes ( a very subjective process) and doing the same for granules, we found the average dark lane intensity to be about 0.72 of the average granule intensity.

\section{Lifetime of Granules}

The lifetime of granules determined by the half-life of the cross-correlation coefficient is about $5 \mathrm{~min}$ (Leighton, 1957). Figure 1 shows the cross-correlation curve of our data further from the spot, which yields the same result. We could not detect any 5-min oscillation pattern in the cross-correlation curve (Figure 1). 


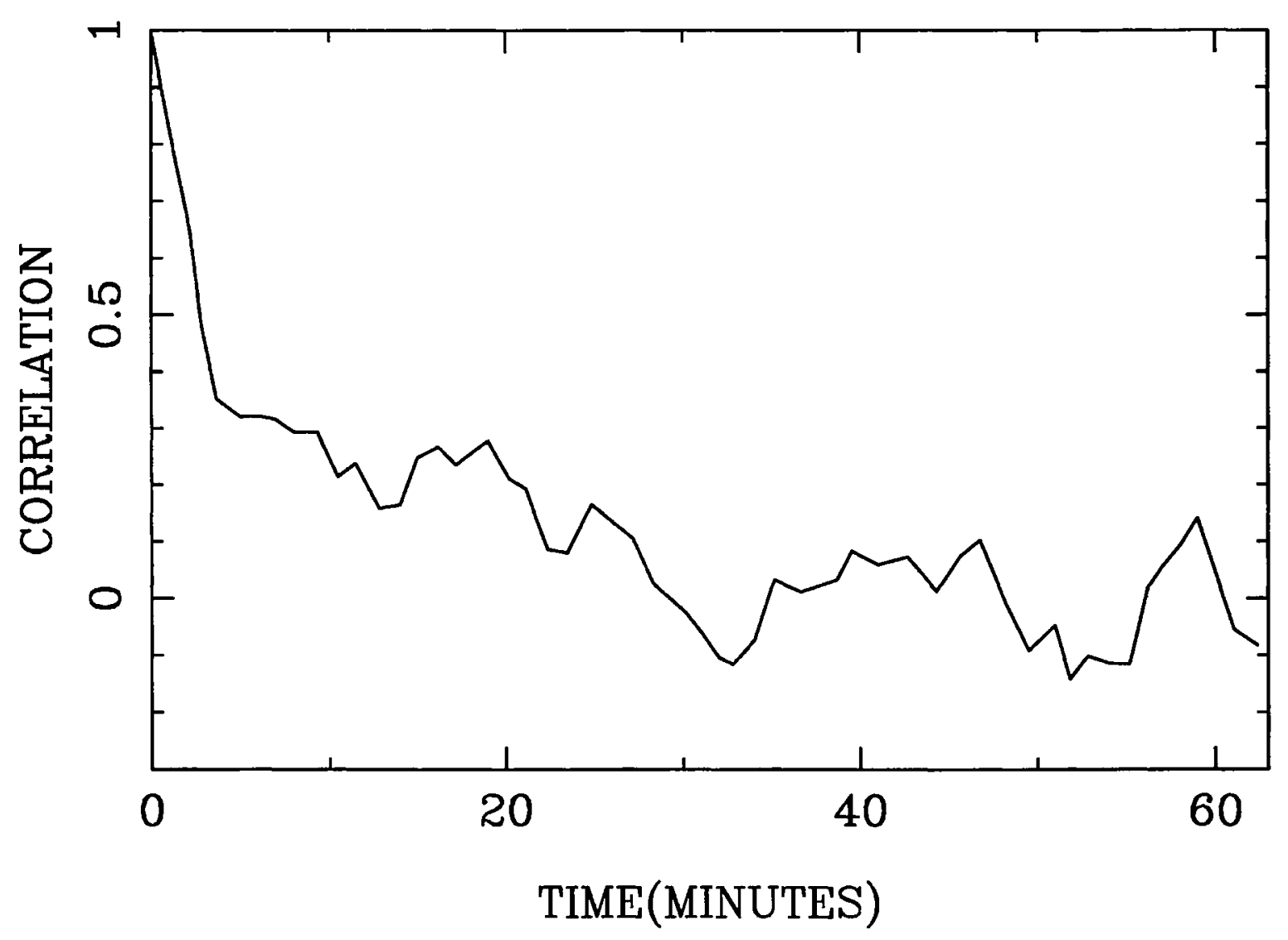

Fig. 1. Cross-correlation curve of region 1, a granular region outside the sunspot.

The decay of cross-correlation measures the change in shape of the granule, but much of this is due to rapid fluctuation in the dark lanes. A granule which started at zero size, grew to maximum in $8 \mathrm{~min}$, and disappeared in the next eight would indeed have an $e$-folding cross-correlation coefficient around $5 \mathrm{~min}$. By tracking the evolution of individual granules, we obtained a longer lifetime for granules. We divided granules into three groups, small $\left(\leq 0.5^{\prime \prime}\right)$, medium $\left(0.5^{\prime \prime}\right.$ to $\left.1.5^{\prime \prime}\right)$ and large $\left(\geq 1.5^{\prime \prime}\right)$. The average lifetimes of granules in these three groups are: $10.7 \pm 2.5 \mathrm{~min}$ for small granules; $16.9 \pm 9.7 \mathrm{~min}$ for medium granules; $21.8 \pm 5.3 \mathrm{~min}$ for large ones. Averaging over all the granules in these groups gives the lifetime of $16.4 \pm 8.3 \mathrm{~min}$. The distribution of granule lifetimes is shown in Figure 2. Muller (1985) discusses this point and notes that Simon (1967) and Mehltretter (1978) define the lifetime as twice the decay time of the surviving granules from a 'master frame' obtaining a lifetime of $16 \mathrm{~min}$. The greater lifetime for larger granules is not surprising.

We must remember that the granules are not discrete elements, but the tops of convective cells. The lifetime is the duration of a certain pattern of flow, and the exploding granule is a spreading of the flow pattern over an adjacent area.

We performed a three-dimensional FFT for granule data in the region further from the sunspot. The results were collapsed to a two-dimensional $k-\omega$ diagram. No ridges were found in the $k-\omega$ diagram. This is in accord with the absence of 5-min oscillation effects. We believe they are absent because our field of view is so small. 


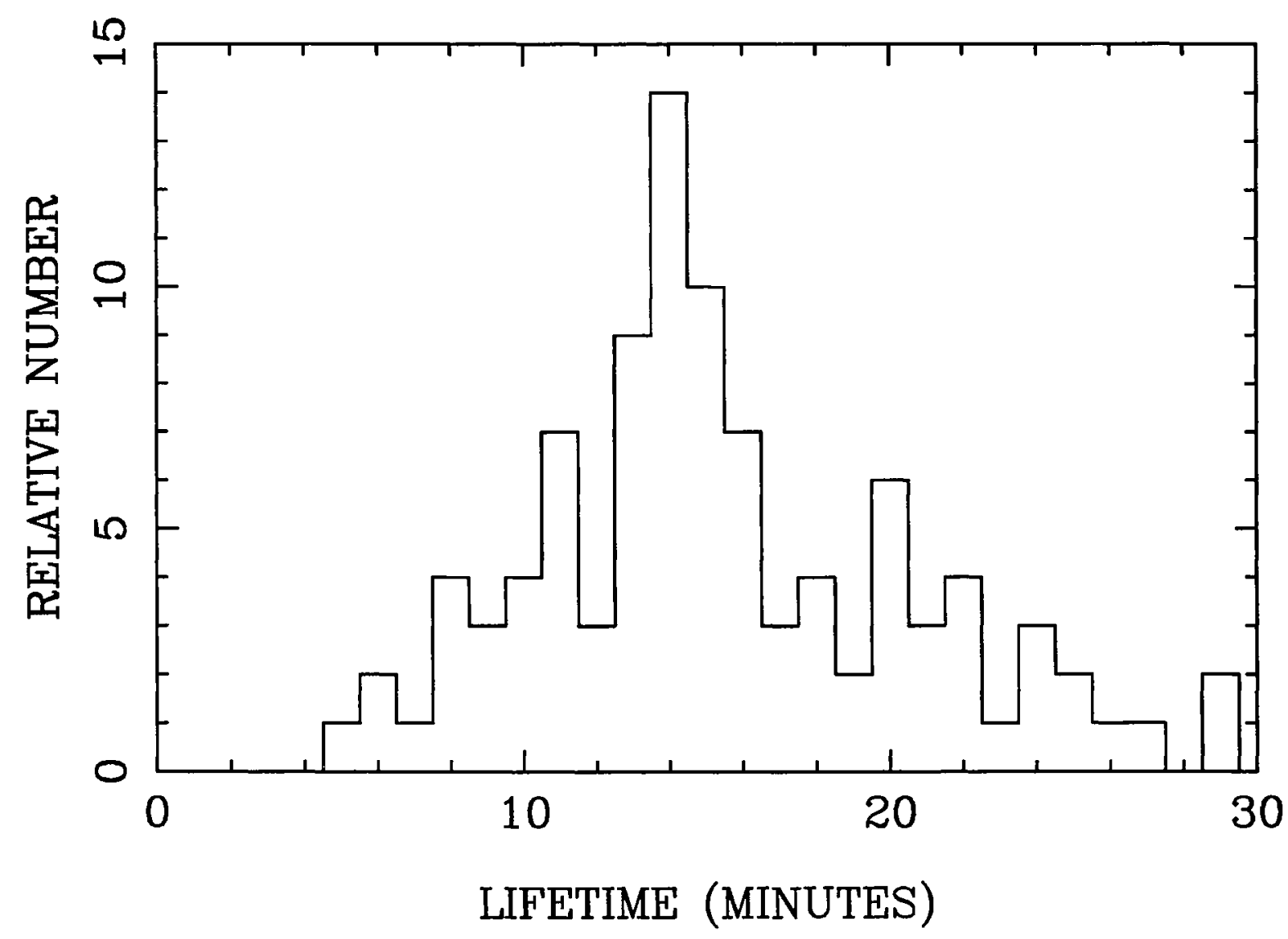

Fig. 2. A histogram showing the distribution of granulation lifetime.

\section{Exploding Granules}

Following the technique used by Title et al. (1986), we constructed 'time slice' images to study the properties of exploding granules. The expanding velocity of exploding granules is found to be 1.0 to $1.5 \mathrm{~km} \mathrm{~s}^{-1}$. After studying the movie for region 1 , we found that on the average $41 \%$ of the area is hit at least once by exploding granules in $60 \mathrm{~min}$. Assuming the average lifetime of granules is $15 \mathrm{~min}, 10 \%$ of granules are hit by exploding granules at least once during their lifetime. We also found that $2 \%$ of granules exploded.

A very interesting phenomenon is a big 'exploding' event that occurred during the observation. Figure 7 (which will be discussed in detail later) shows a sequence of frames of the region centered on the sunspot. The big 'exploding' event occurred near the top-right corner of the field of view, as marked by ' $\mathrm{g}$ '. An elongated large granule of $2^{\prime \prime}$ by $4^{\prime \prime}$ formed at 18:10 UT, exploded at 18:17 UT, and erupted again at $18: 20$ UT, reaching a size $5^{\prime \prime}$ by $6 "$, about a fourfold increase. The outward speed is $1 \mathrm{~km} \mathrm{~s}^{-1}$. This is the only feature in our data with the scale of the mesogranulation suggested by November et al. $(1981,1982)$. 

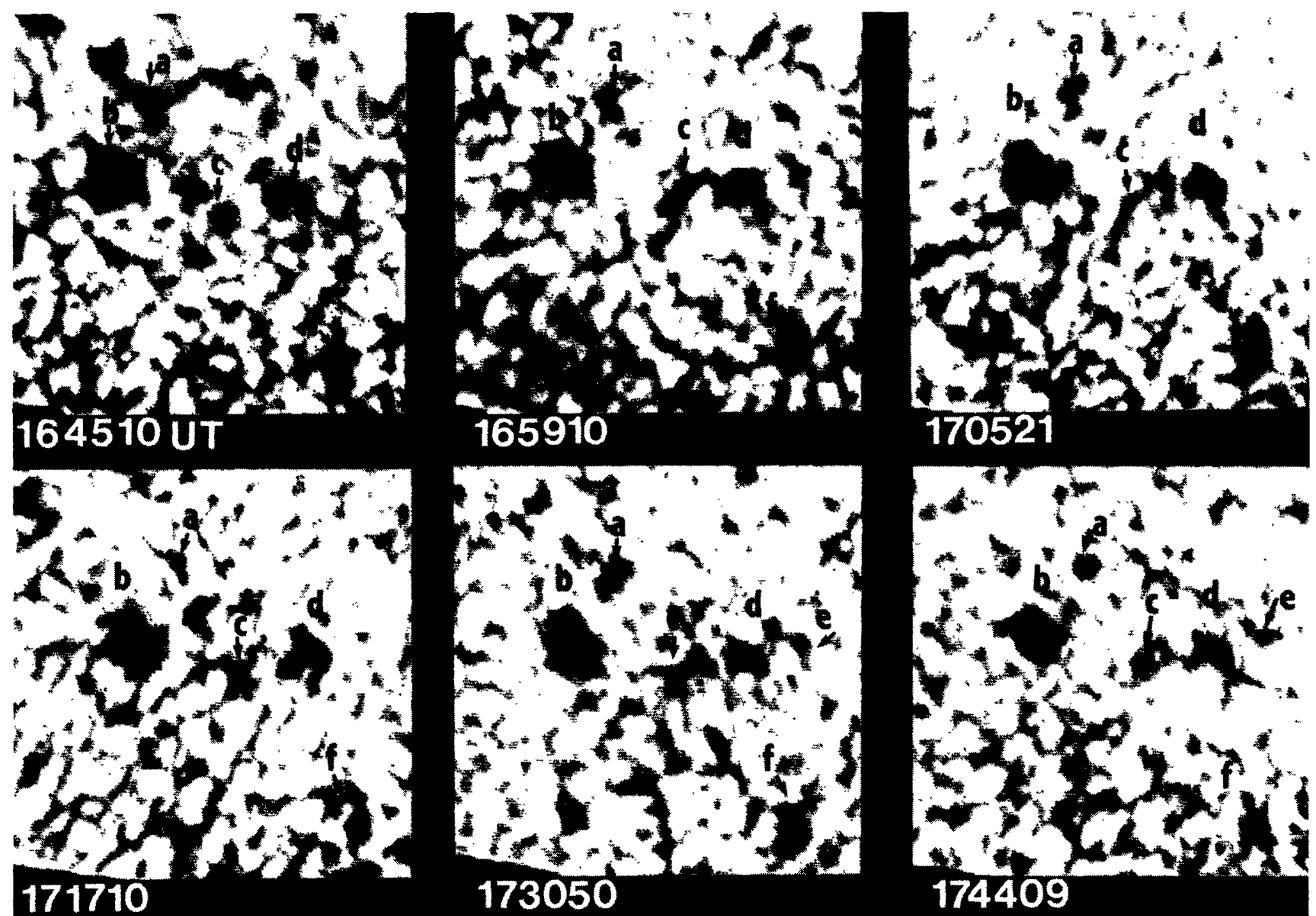
173050

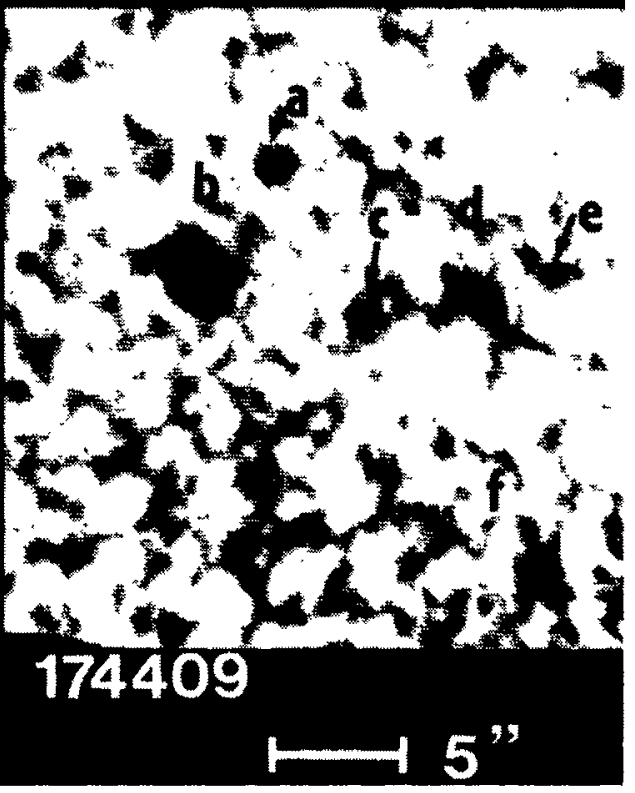

Fig. 3. A sequence of white-light images showing region 1, the region outside the sunspot. 'a', ' $b$ ', 'c', ' $d$ ', ' $e$ ', and ' $f$ ' are six pores; most of them show rapid 


\section{Pores}

We found that several pores changed their sizes and contrasts rapidly during the one-hour observation. Unfortunately, the smallest pores are not readily distinguishable from the largest dark lanes, but we used the distinction that the lanes varied rapidly in less than a minute, while pores were more stable.

In Figure 3, we show a sequence of pictures that contain 6 pores: ' $a$ ', 'b', 'c', 'd', 'e', and ' $f$ '. Of these only ' $b$ ' is substantially larger than a granule, and only ' $b$ ' is relatively unchanged. Pore ' $a$ ' forms during the run, pore ' $c$ ' changes strongly and pore ' $e$ ' forms at the very end in a few minutes. Pore ' $d$ ' undergoes sizeable changes. Pore ' $f$ ' remained in the field of view during the second run; it is also marked in Figure 7. This pore is observed to wax and wane with a 30 min period. It is almost gone by the end of the second sequence. The satellite spots in the second run are also seen to change.

Figure 4 shows the variation of integrated darkening of pore ' $f$ ', which could be followed on both videotapes. Its relative intensity oscillated four times with maxima roughly $30 \mathrm{~min}$ apart. The integrated darkness is the fractional decrease in brightness times the area in $\operatorname{arc~} \sec ^{2}$. It is measured by integrating the contrast over the area of the pore. The contrast is calibrated by a neutral density filter on the corner of the image. Figure 5 shows the integrated darkness of the pore 'e' which forms in less than $20 \mathrm{~min}$.

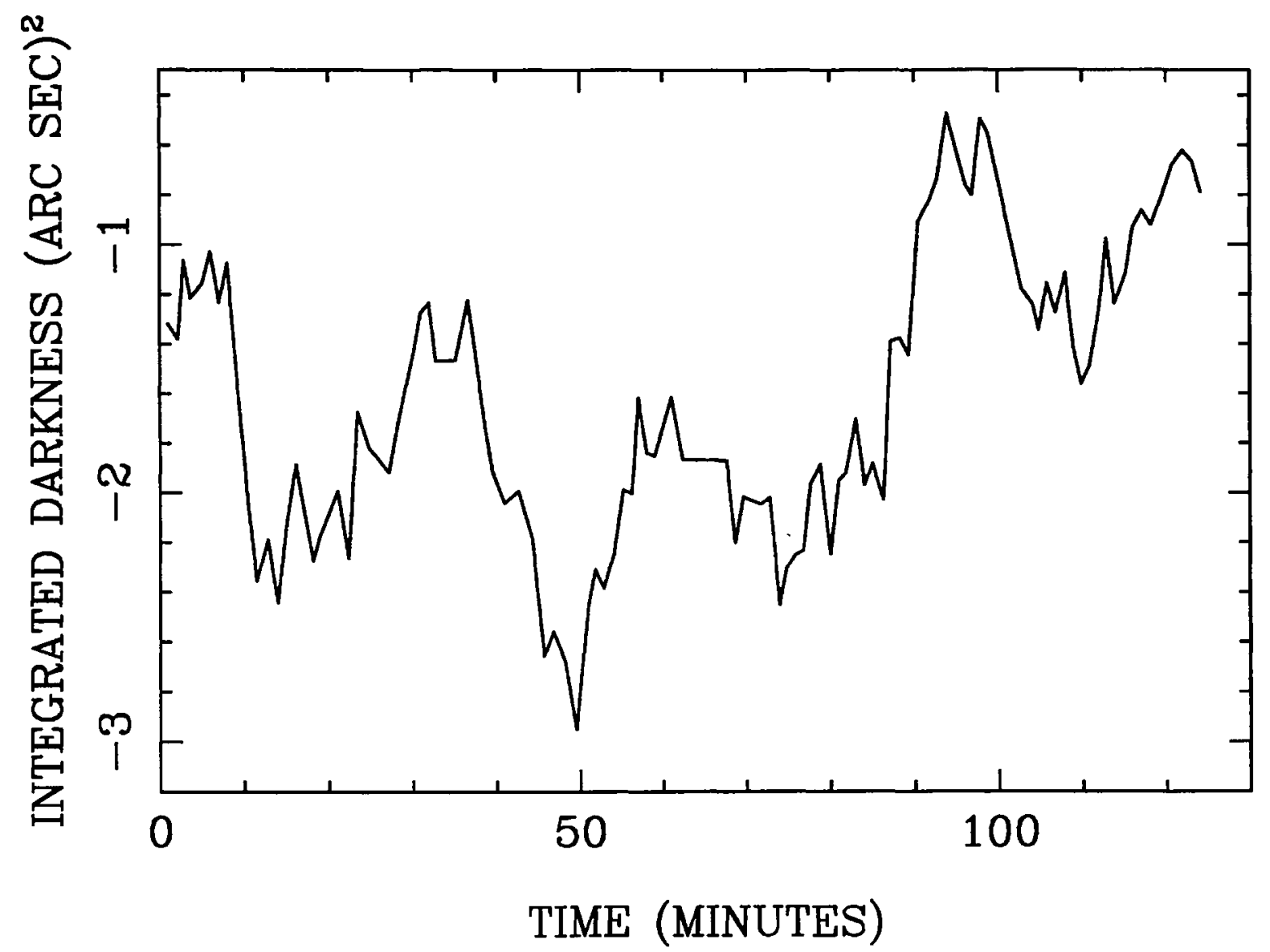

Fig. 4. An example of a rapidly changing pore. The curve shows the integrated darkness of the pore as a function of time. The integrated darkness appears to 'oscillate' with a period of $30 \mathrm{~min}$. 


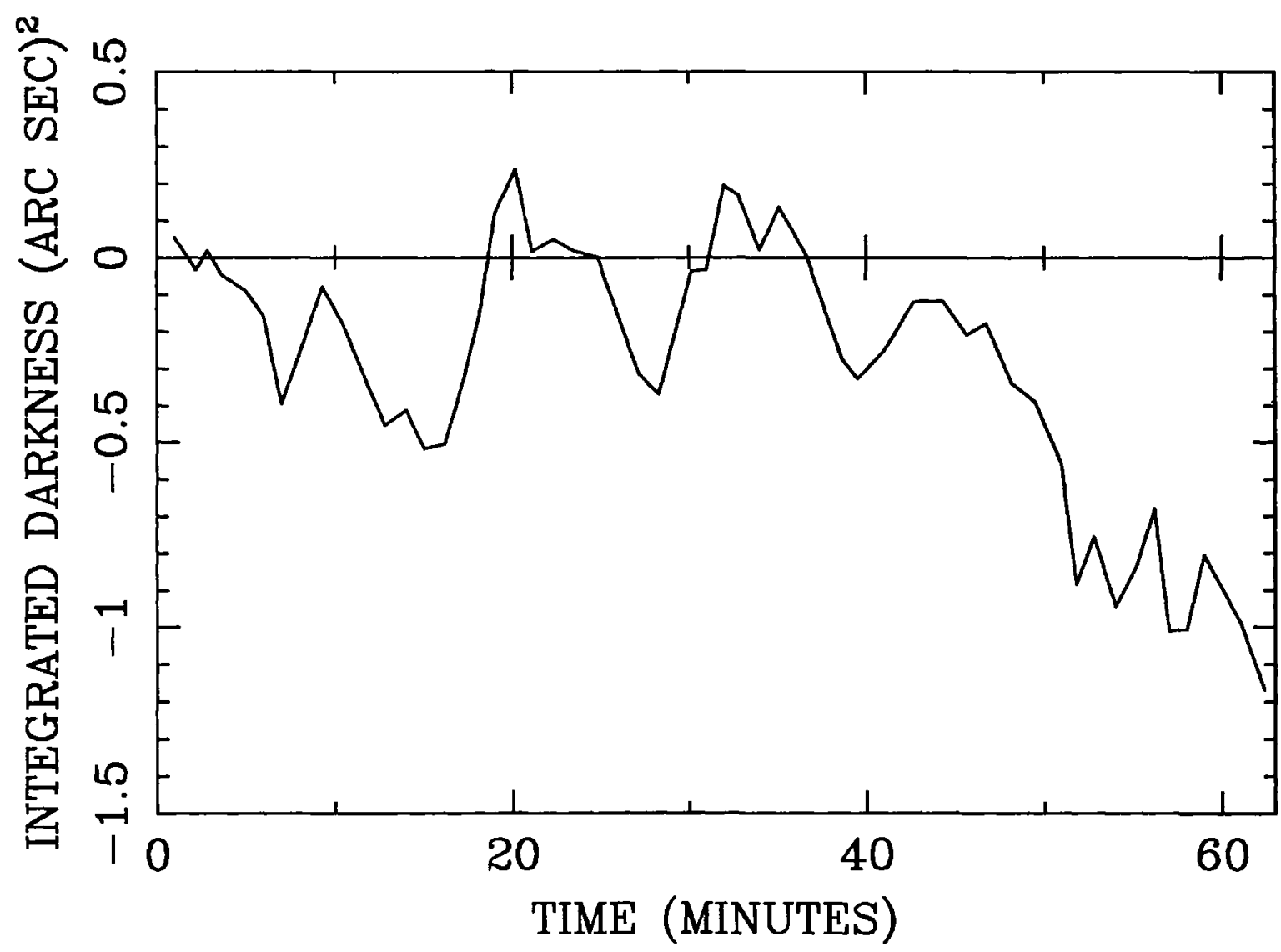

Fig. 5. Another example of a rapidly changing pore. The pore formed in less than $20 \mathrm{~min}$.

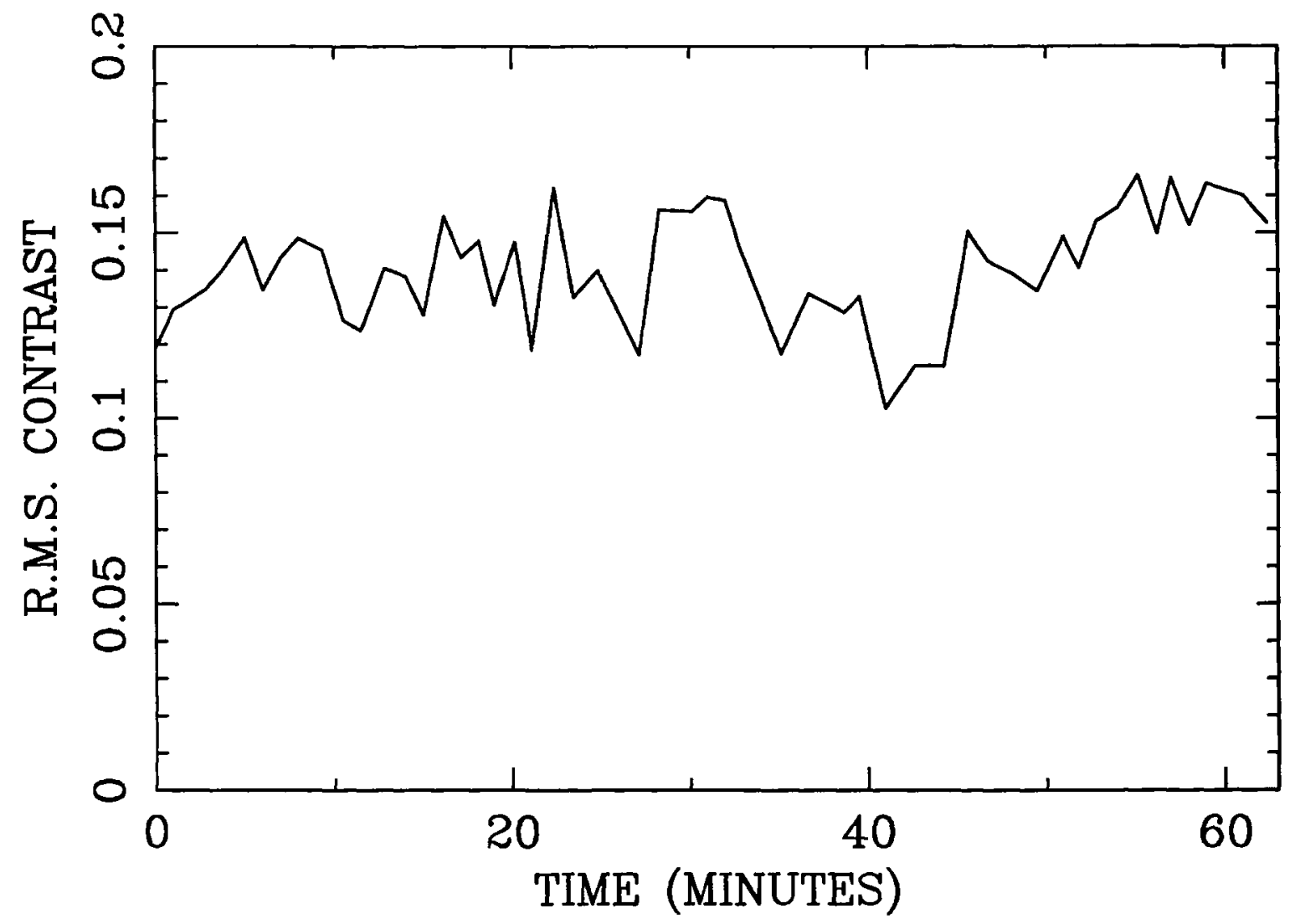

Fig. 6. R.m.s. contrast as a function of time. We use this curve as a seeing index. 
We know that the changes in the pores are real and not due to seeing because we have videotapes with 30 frames $^{-1}$ which show the area change is somewhat slower than the seeing and confirmed by several frames.

Another way to test whether the change of pore intensity is due to seeing variation, is to plot the r.m.s. contrast of the image as a function of time. We assume that the r.m.s. contrast is a good indicator of seeing. Such a curve is plotted in Figure 6. Obviously, the variation of pore intensity is not due to the seeing variation.

All the pores fluctuate in brightness on a scale of one or two equivalent arc sec, and this appears due to granule activity. For a small pore less than 1 arc sec square this may represent life or death, while for the larger ones the effect is smaller. The cooling and heating times observed are still long compared to the values computed by Chou (1987), but in this case we have no idea of the underlying magnetic changes. It appears from the foregoing that the granule lifetime is the important cooling time for small pores.

\section{Penumbral Flows}

Our second video sequence (Figure 7) shows a large stable sunspot. Both bright and dark structures in the penumbra move inward with average velocity $0.5 \mathrm{~km} \mathrm{~s}^{-1}$. These structures usually do not die but disappear into the umbra where the image is underexposed.

The penumbral motions are shown by the local correlation tracking map (Figure 8). The local correlation technique has been discussed by November et al. (1986). The results obtained by correlation tracking agree with the flow pattern studied visually. In Figure 7, we marked three penumbra fibrils. ' 1 ' and ' 2 ' are two dark fibrils and ' 3 ' is a bright one. All of these fibrils move inward with a speed around $0.5 \mathrm{~km} \mathrm{~s}^{-1}$. The window size used in correlation tracking is $1.5^{\prime \prime}$ by $1.5^{\prime \prime}$. The average amplitude of inflows is $0.5 \mathrm{~km} \mathrm{~s}^{-1}$. The motions are easily followed, as we see in Figure 8. It is hard to understand the relation between this flow and the Evershed flow, which is outward. The only possible difference might be height, since the latter is observed near the limb. But the height difference appears small for such a striking reversal. Another possibility is that the Evershed flow is outside the penumbra, associated with the surrounding outflow. The evidence, however, strongly supports the limitation of the Evershed flow to the penumbra.

Both examination of the films and the correlation tracking reveal important outflow around the sunspot, starting from the outer edge of penumbra. The maximum outflow speed is $1.5 \mathrm{~km} \mathrm{~s}^{-1}$. Average speed is $1.0 \mathrm{~km} \mathrm{~s}^{-1}$. The outflow may be the same as the well-known 'moat' phenomenon (Vrabec, 1974). The velocity is larger than that of the penumbral inflow. The outflow obtained by correlation tracking is also shown in Figure 8. Another aspect of the outflow is the appearance of transient dark outflow clouds a few arc sec across. These are always aligned radially from the spot and last about $20 \mathrm{~min}$.

As discussed by Shine et al. (1986), some occasional bright structures ('streakers') appear to be ejected outward from the penumbra. Those jets-like streakers last about 


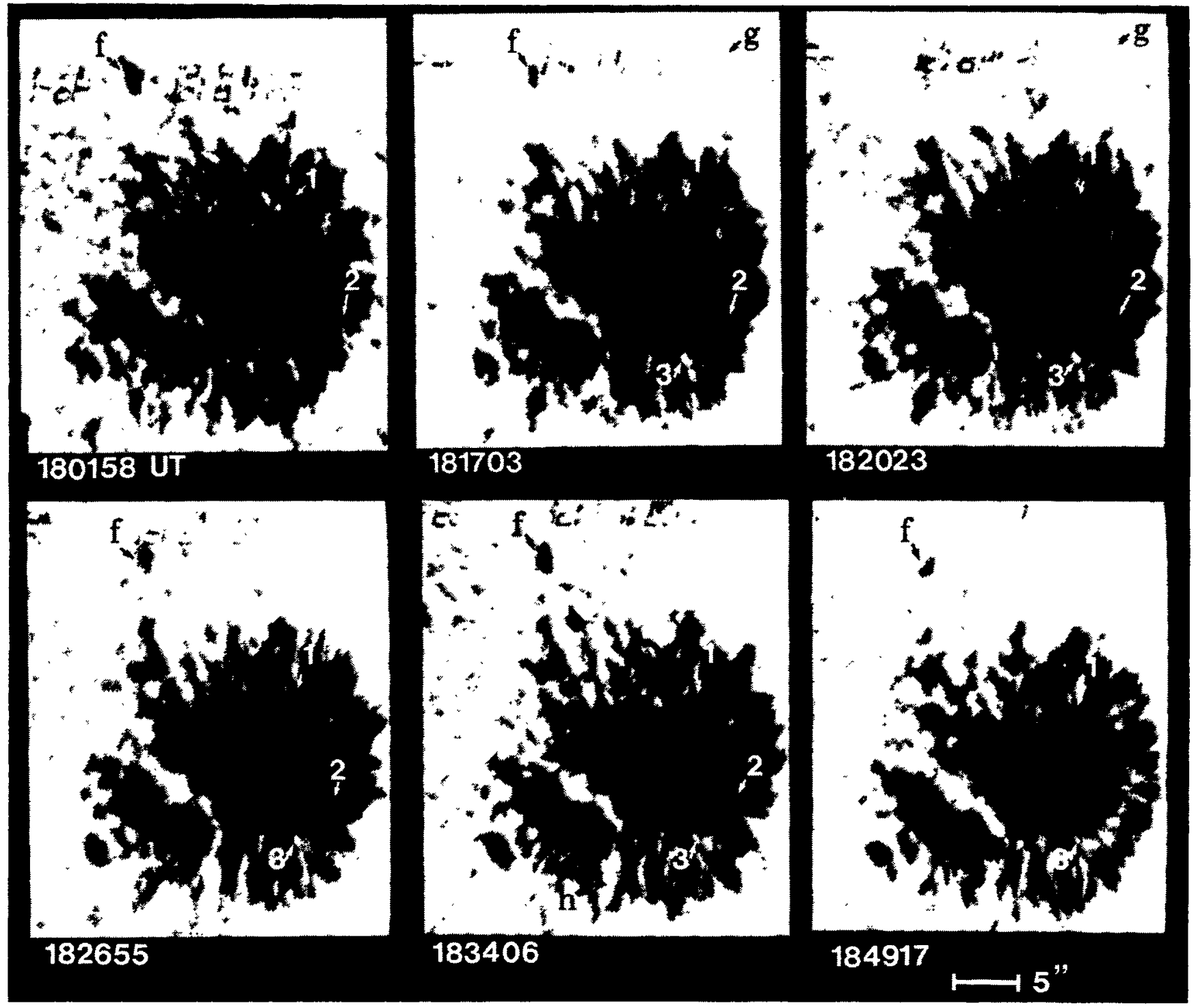

Fig. 7. A sequence of white-light images showing region 2 , the region centered on the sunspot. ' 1 ', ' 2 ', and ' 3 ' are three penumbra fibrils which move towards the umbra. ' $f$ ' is the same pore ' $f$ ' shown in Figure 3. ' $g$ ' shows a big exploding event. ' $h$ ' is a prominent streaker.

10 to $20 \mathrm{~min}$. Their velocity is hard to measure. We found one prominent streaker (marked by ' $h$ ' in Figure 7) lasting 20 min and 1.2 times brighter than the penumbra. Whether it bears any relation to white light flares or not we do not know; our $\mathrm{H} \alpha$ is inadequate to check for flare brightening in this case.

At the upper left of the large spot and the light bridge between two satellite spots, we find what has been referred to as umbral dots. So far as we can tell, the dots are indistinguishable from the normal granulation, except that they are isolated from one another. They do not, however share the inward streaming of the penumbral granules.

We also applied the local correlation technique to region 1, the region further from the spot. Some converging and diverging flow patterns are found. The cork movies show results quite similar to the SOUP data (Title et al., 1986). We found 10 'sinks' in a field of view of $40^{\prime \prime}$ by $40^{\prime \prime}$. Since our field of view was about the size of a supergranule cell, 


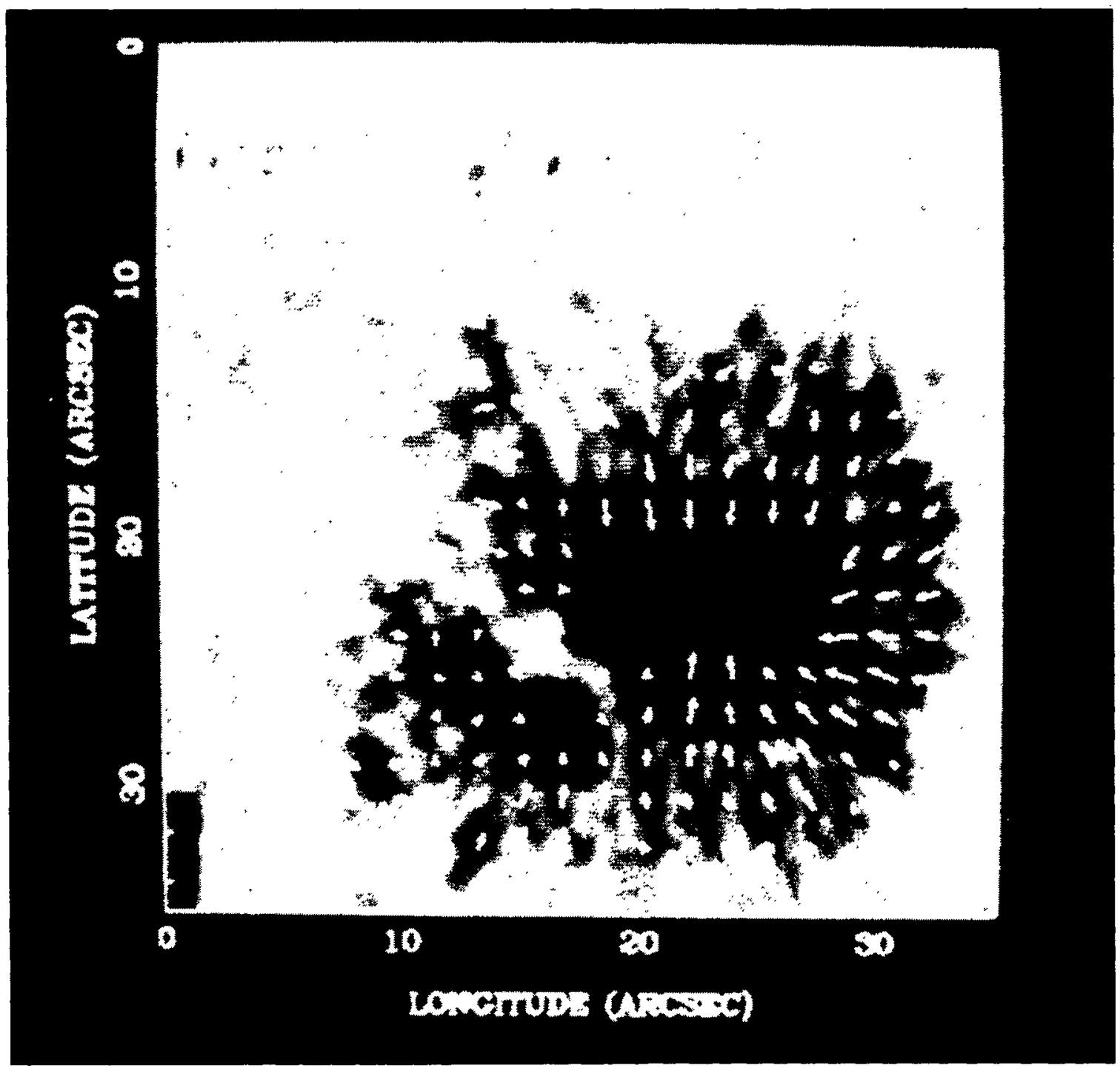

Fig. 8. Velocity map derived by local correlation tracking for the region centered on the spot (region 2).

and we did not have corresponding VMG or $\mathrm{Ca} \mathrm{K}$ observation, it is hard to decide if the flow is the supergranule flow and converging areas are located at the boundaries of supergranules.

\section{Summary}

After studying high quality granulation movies in detail, we obtained the following results:

(1) The average lifetime of granules is $16 \mathrm{~min}$. Larger granules have longer lifetimes.

(2) Two percent of granules explode. The probability that a granule is hit by exploding granules at least once is $10 \%$. We also observed a big exploding event covering about 10 granules.

(3) Many small pores change their size and darkness in less than a time-scale of 
$60 \mathrm{~min}$. The integrated darkness of one pore appears to be oscillating with a period of about $30 \mathrm{~min}$.

(4) There are clear inflows of both bright and dark penumbra fibrils towards the umbra, from the outer edge of penumbra. We also confirmed the outflow from the outer edge of penumbra. This flow pattern may be related to the moving magnetic features and the moat structure surrounding the sunspots (Sheeley and Bhatnagar, 1971; Harvey and Harvey, 1973; Vrabec, 1974). It also confirms the study by Muller and Mena (1987).

\section{Acknowledgements}

We are indebted to Bill Marquette and Randy Fear for their assistance in obtaining the data. We are indebted to the referee for a number of valuable comments. This work is supported by NSF under grant ATM-8513577 and NASA under grant NGL 05002034.

\section{References}

Bray, R. J., Loughhead, R. E. and Durant, C. J.: 1984, The Solar Granulation, 2nd ed., Cambridge Univ. Press, London.

Chou, D.: 1987, Astrophys. J. 312, 955.

Harvey, K. and Harvey, J.: 1973, Solar Phys. 28, 61.

Kitai, R. and Kawaguchi, I.: 1979, Solar Phys. 64, 3.

Kawaguchi, I.: 1980, Solar Phys. 65, 207.

Leighton, R. B.: 1957, Publ. Astron. Soc. Pacific 69, 411.

Mehltretter, J. P.: 1978, Astron. Astrophys. 62, 311.

Muller, R.: 1985, Solar Phys. 100, 237.

Muller, R. and Mena, B.: 1987, Solar Phys. 112, 295.

November, L. J., Toomre, J., and Gebbie, K. B.: 1981, Astrophys. J. 245, L123.

November, L. J., Toomre, J., Gebbie, K. B., and Simon, G. W.: 1982, Astrophys. J. 258, 846.

November, L., Simon, G., Tarbell, G., Title, A., and Ferguson, S.: 1986, Theoretic Problem in High Resolution Solar Physics, NASA CP-2483, p. 121.

Oda, N.: 1984, Solar Phys. 93, 243.

Roudier, Th. and Muller, R.: 1987, Solar Phys. 107, 11.

Sheeley, N. R. and Bhatnagar, A.: 1971, Solar Phys. 19, 338.

Shine, R. A., Title, A., Tarbell, T., and the SOUP Team: 1986, Theoretic Problem in High Resolution Solar Physics, NASA CP-2483, p. 133.

Simon, G. W.: 1967, Z. Astrophys. 65, 345.

Simon, G. W., Title, A. M., Topka, K. P., Shine, R. A., Ferguson, S. H., Zirin, H., and the SOUP Team: 1988, Astrophys. J. 327.

Title, A., Tarbell, T., and the SOUP Team: 1986, Theoretic Problem in High Resolution Solar Physics, NASA CP-2483, p. 55.

Vrabec, D.: 1974, in R. G. Athay (ed.), 'Chromospheric Fine Structure’, IAU Symp. 56, 201. 